Proc. Indian Acad. Sci. (Earth Planet. Sci.), Vol. 100, No. 3, September 1991, pp. 267-280.

(C) Printed in India.

\title{
Gravity induced near-surface stresses in long-symmetric ridge-valley systems
}

\author{
P MANDAL and R N SINGH \\ National Geophysical Research Institute, Hyderabad 500007, India \\ MS received 19 June 1991; revised 20 August 1991
}

\begin{abstract}
Patterns of near-surface gravity and tectonically-induced stresses within ridgevalley systems greatly illuminate our understanding of important geodynamic processes as well as design of experiments to elucidate them. This paper presents analytical results derived for a number of geometrical configurations and range of mechanical properties of a ridge-valley system using the elastic solution of McTigue and Mei. The study reveals (i) the presence of non-zero compressive stresses near the ridge crests, which decrease with increasing Poisson's ratio $(\mu)$ and reduce to zero at ridge crests for $\mu=0.5$ and that (ii) the central tensional regime characterizing a valley becomes narrower due to the presence of two ridges, and decreases with increasing $\mu$, becoming compressive at depth. For all geometrical parameters considered, all components of stress show concentration at the outer flanks of the ridges and increase with depth approaching a standard state of stress.
\end{abstract}

Keywords. Stresses; ridge-valley; topography.

\section{Introduction}

Surface topographic features are associated with stresses inside the lithosphere, the affected region depending on the length scale of topography. Knowledge of such stress patterns can shed significant light on several geodynamical situations such as geomorphic evolution, isostasy, fracture propagation involved in magma dynamics and hydraulic fracturing. Stresses associated with topography have been calculated earlier in the context of isostasy and these are useful for large regional scale lithospheric studies. Jefferys (1976) analytically derived stresses in an elastic half-space caused by a topographic load representing it by the normal pressure source at its upper surface. An elastic thin-plate model has also been used to determine such stresses (Brotchie and Silvester 1969; Lambeck and Nakiboglu 1980).

Calculations of stresses within a topography and near it have also been made mainly using numerical methods (Harrison 1976; Strugel et al 1976; Jaeger and Cook 1976). Analytical solutions for gravity-induced stresses have been given using conformal mapping by Savage et al (1985) and Savage and Swolfs (1986) expanding the work of Akhpatelov and Ter-Martirosyan (1971). These solutions are exact, but have limited applications due to difficulty in obtaining the conformal transformation of the realistic topographic features. Thin topographic features (having small slopes) occur on the earth's surface in abundance and stresses associated with them have been calculated by McTigue and Mei (1981) using an approximate analytical procedure. This technique has been used to estimate gravity-induced stresses associated with topographic irregularities (e.g. ridge, valley, hill etc.) with small 


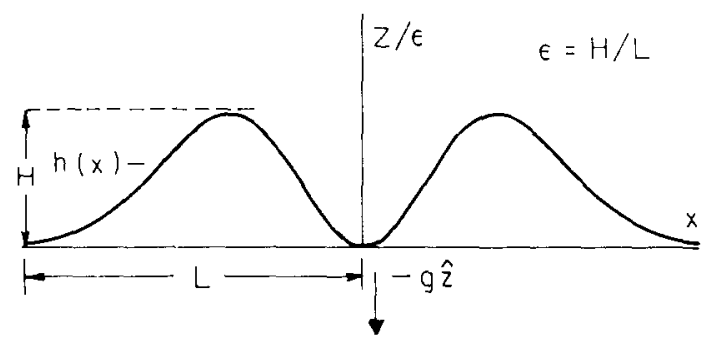

Figure 1. Coordinate system and parameters used in stress computations.

characteristic slopes and for calculating stresses associated with thrust propagation (Panian and Pilant 1990).

In the present paper, we report the determination of stresses for a topography consisting of ridge-valley structures (figure 1) using the above mentioned method. Such structures frequently occur in the mid-oceanic crustal regions. Near-surface stresses of these topographies are required to understand the seismicity and magma transport phenomena. These results are approximate solutions of the order of $\varepsilon^{2}$, where $\varepsilon$ is a characteristic topographic slope which should be very small $(\varepsilon \ll 1 \cdot 0)$. A brief survey of the observed topography over oceanic ridge-valley structures shows that this is not a severe limitation. Even areas of higher relief may be treated within acceptable bounds. For example, the typical slope of a slow spreading mid-oceanic ridge with $1.5-3 \mathrm{~km}$ deep and $10-15 \mathrm{~km}$ wide rift valley, as considered by Chen and Morgan (1990), has $\varepsilon=0.15-0.20$ giving an error of $2.25-4 \%$. Similarly, a typical slope in the south-east ridge of Indian ocean with $1-1.5 \mathrm{~km}$ deep and $200 \mathrm{~km}$ wide valley shows $\varepsilon=0.01-0.015$ giving an error of $0.01-0.02 \%$.

\section{Mathematical formulation and solutions}

We first review the method of McTigue and Mei (1981) for solving plane elasto-static problems. A perturbation scheme is used to obtain integral representations (accurate to first order) for gravity-induced near-surface stresses within different topographic reliefs characterized by small slopes. A two-dimensional homogeneous elastic half-space model with arbitrarily-shaped free surface is considered. A Cartesian coordinate system is defined with $z$ as a vertical axis positive upwards and a constant body force, $g \hat{z}$, directed vertically downward (figure 1 ).

The stresses $\bar{\sigma}$ are calculated using the equation of equilibrium and the compatibility condition as given below:

$$
\begin{aligned}
& \nabla \cdot \bar{\sigma}-g \hat{z}=0, \\
& \nabla^{2}\left(\sigma_{x x}+\sigma_{z z}\right)=0,
\end{aligned}
$$

where, $\sigma_{x x}$ and $\sigma_{z z}$ are the normal components of the stress acting in $x$ and $z$ directions respectively. The boundary condition at the upper irregular surface is:

$$
\bar{\sigma} \cdot \hat{n}=0 \text { at } z=\varepsilon h(x),
$$

where $\hat{n}$ and $\hat{z}$ are the unit normal to irregular surface and the unit vector in the direction of acceleration due to gravity $(\hat{g})$ respectively. For structures below water, this 
condition (3) should be changed to include the effects of water load. These equations are non-dimensionalized using the following scheme:

$$
x=L x^{*}, z=L z^{*}, \sigma=\rho g H \sigma^{*} \text { and } H(x)=L h\left(x^{*}\right) .
$$

We then get,

$$
\begin{aligned}
& \nabla \cdot \sigma^{*}-\frac{1}{\varepsilon} \hat{\jmath}=0, \\
& \nabla^{2} \cdot\left(\sigma_{x x}^{*}+\sigma_{z z}^{*}\right)=0, \\
& \sigma^{*} \cdot \hat{n}=0 \text { on } z^{*}=\varepsilon h\left(x^{*}\right) .
\end{aligned}
$$

The stresses can be written in terms of Airy stress function $\phi(x, z)$ as (Savage et al 1985):

$$
\begin{aligned}
\sigma_{x x} & =\frac{\partial^{2} \phi(x, z)}{\partial^{2} z}+\frac{\mu}{(1-\mu)} \frac{z}{\varepsilon} \\
\sigma_{z z} & =\frac{\partial^{2} \phi(x, z)}{\partial^{2} x}+\frac{z}{\varepsilon} \\
\sigma_{x z} & =-\frac{\partial \phi^{2}(x, z)}{\partial x \partial z}
\end{aligned}
$$

The equilibrium equation (1) is satisfied by equations (7), (8) and (9). The second terms on the right side of (7) and (8) represent the standard states of stresses for a flat-lying hoizontal region under gravity (Jaegar and Cook 1976). Further, according to compatibility condition (2), $\phi(x, z)$ satisfies the biharmonic equation

$$
\nabla^{4} \phi(x, z)=0
$$

For small values of $\varepsilon$, McTigue and Mei (1981) obtained the following solution of (10) using the boundary condition (6)

$$
\begin{aligned}
& \sigma_{z z}=\frac{z}{\varepsilon}-h(x)+O\left(\varepsilon^{2}\right), \\
& \sigma_{x x}=\frac{\mu}{(\mu-1)} \frac{z}{\varepsilon}-h(x)+\varepsilon \frac{1}{\pi} \frac{(2-3 \mu)}{(2-2 \mu)} \int_{-\infty}^{+\infty} \bar{h}^{2}|\xi| \exp (-i \xi x) \mathrm{d} \xi \\
& -z \frac{1}{\pi} \int_{-\infty}^{+\infty} \bar{h}|\xi| \exp (-i \xi x) \mathrm{d} \xi+O\left(\varepsilon^{2}\right), \\
& \sigma_{x z}=-\varepsilon \frac{(2-3 \mu)}{(2-2 \mu}\left(h^{2}\right)_{, x}+z(h)_{, x}+O\left(\varepsilon^{2}\right),
\end{aligned}
$$

where $\bar{h}$ and $\bar{h}^{2}$ are the Fourier transforms of $h(x)$ and $h^{2}(x)$, respectively. The effect of the distributed shear is represented by terms quadratic in $h$ and the distributed normal load is represented by terms multiplied by $z$.

McTigue and Mei (1981) also extended their work for far-field tectonic forces (compression or tension) on the gravitationally-induced stress field. Their results for 
stress distribution due to far-field tectonic stresses are:

$$
\begin{aligned}
& \sigma_{z z}^{t}=0+O\left(\varepsilon^{2}\right), \\
& \sigma_{x x}^{t}=R\left(1-\varepsilon \frac{1}{\pi} \int_{-\infty}^{+\infty} \bar{h}|\varepsilon| \exp (-i \xi x) \mathrm{d} \xi\right)+O\left(\varepsilon^{2}\right), \\
& \sigma_{x z}^{t}=\varepsilon R \frac{\partial h}{\partial x}+O\left(\varepsilon^{2}\right),
\end{aligned}
$$

where $R$ is the ratio of the tectonic stress to the gravitational stress $\left(R=\sigma_{\infty} / \rho g H\right)$; $R$ is positive for regional tension and negative for regional compression. Equations (11), (12) and (13) can be added with (14), (15) and (16) respectively to obtain the stresses due to combined gravity and tectonic forces.

We now apply the above formalism for ridge-valley systems represented by,

$$
h(x)=\left(x^{2} / b^{2}\right) \exp \left[\left(b^{2}-x^{2}\right) / b^{2}\right] .
$$

Using (12), (15) and (17), the horizontal normal stress is obtained as:

$$
\begin{aligned}
\sigma_{x x}= & \frac{\mu}{\mu-1} \frac{z}{\varepsilon}-h(x)+\varepsilon \frac{(2-3 \mu)}{(1-\mu)} \frac{1}{16} \sqrt{2} \pi^{5 / 2} a\left(3 I_{3}-\frac{3}{2 a} I_{2}+\frac{1}{16 a^{2}} I_{1}\right) \\
& -2 z \frac{1}{\sqrt{\pi} a} I_{4}+R\left(1-2 \varepsilon I_{4}\right)+O\left(\varepsilon^{2}\right),
\end{aligned}
$$

where,

$$
\begin{aligned}
& I_{1}(x)=\left(-32 \sqrt{2} a^{5 / 2}\right)\left[-8+36 a^{2} x^{4}-16 a^{2} x^{4}+\operatorname{Frn} 2(x)(60 b x\right. \\
&\left.\left.-160 b^{2} x^{3}+64 b^{3} x^{5}\right)\right], \\
& I_{2}(x)= 8 \sqrt{2} a^{3 / 2}\left[1-4 a x^{2}+4 a x\left(4 a x^{2}-3\right) \operatorname{Frn} 2(x)\right], \\
& I_{3}(x)= 2 \sqrt{2}[1-4 a x \operatorname{Frn} 2(x)], \\
& I_{4}(x)= {\left[2 a x^{2}+4 a x\left(1-a x^{2}\right) \operatorname{Frn} 1(x)\right], } \\
& R=\sigma_{\infty} / \rho g H .
\end{aligned}
$$

Dwason's integrals are defined as

$$
\begin{aligned}
& \operatorname{Frn} 1(x)=\exp \left(-a x^{2}\right) \int_{0}^{x \sqrt{a}} \exp \left(t^{2}\right) \mathrm{d} t \\
& \operatorname{Frn} 2(x)=\exp \left(-2 a x^{2}\right) \int_{0}^{x \sqrt{2 a}} \exp \left(t^{2}\right) \mathrm{d} t
\end{aligned}
$$

Similarly, using equations (11), (13), (14), (16), and (17), vertical normal and horizontal shear stresses are obtained as

$$
\begin{aligned}
& \sigma_{z z}=\frac{z}{\varepsilon}-\frac{x^{2}}{b^{2}} \exp \left(\left(b^{2}-x^{2}\right) / b^{2}\right)+O\left(\varepsilon^{2}\right), \\
& \sigma_{x z}=-\varepsilon \frac{(2-3 \mu)}{(2-2 \mu)} \frac{\partial h^{2}}{\partial x}+z \frac{\partial h}{\partial x}+O\left(\varepsilon^{2}\right)+R\left(\varepsilon \frac{\partial h}{\partial x}\right) .
\end{aligned}
$$


The maximum shear $(\tau)$ at any point has been obtained from equations (18), (19) and (20) and is given by:

$$
\tau=\left(\sigma_{x z}^{2}+\frac{1}{4}\left(\sigma_{x x}+\sigma_{z z}\right)^{2}\right)^{1 / 2}
$$

\section{Computational results}

We computed results for several values of the width of ridge-valley systems and Poisson's ratio. The effects of far-fieid stresses are also calculated. These results are discussed in the following.

\subsection{Variation of width of the ridge-valley systems}

Figure 2 shows the stress contour diagram of dimensionless stresses $\sigma_{x x} / \rho g H, \sigma_{z z} / \rho g H$, $\sigma_{x z} / \rho g H$, and $\tau / \rho g H$ for $b=1 \cdot 0$. Stresses are of the order of $\rho g H$. It is clear from figure $2 \mathrm{~b}$ that in the central valley, $\sigma_{x x}$ is tensile and changes to compression on the crest of the ridges. There is a large stress concentration on the outer flanks of the ridges. The vertical normal stress, $\sigma_{z z}$ is compressive and increases with depth with zero value at the surface (figure $2 a$ ). The shear stress term $\sigma_{x z}$ is asymmetric with the central axis of the ridge-valley system (figure $2 \mathrm{c}$ ). At both the ridges and valley, the shear stress is zero and marks a transition from tensile to compressive states. The maximum shear stress $(\tau)$ contour has also been obtained (figure $2 \mathrm{~d}$ ). It shows the concentration of stresses at outer flanks with lesser value at the valley and its adjoining inner flanks.

Figures 3 and 4 illustrate the results for $b=\sqrt{ } 2$ and $1 / \sqrt{ } 3$ respectively. Figure 3 is for a narrow and sharp ridge-valley topography. There is not much change in $\sigma_{x z}$ $\sigma_{z z}$, but the $\sigma_{x x}$ and $\tau$ contours show some changes. $\sigma_{x x}$ is more compressive at the ridge crests and becomes more tensile near the central valley at the surface than the tensile regime of figure $2 \mathrm{~b}$. Similarly maximum shear stress $(\tau)$ contours (figure $3 \mathrm{~d}$ ) has much larger values than in figure $2 \mathrm{~d}$. A smooth and symmetric ridge-valley topography has been shown in figure 4 . It does not show much variation in $\sigma_{x z}$ and $\sigma_{z z}$ contours than the other cases. But $\sigma_{x x}$ contours show greater concentration with a larger value of the stresses at the outer flanks of the ridges. $\sigma_{x x}$ are more compressive at the ridge crests and becomes more tensile on the central valley region than in figures 2 and 3. The maximum shear stress $(\tau)$ contours have shown more concentration with larger magnitude at the outer flanks of the ridges and become nearly zero at the central valley region (figure $4 d$ ).

\subsection{Influence of Poisson's ratio variation}

Figures 5 and 6 show the variation of stress contours due to increase in Poisson's ratios i.e. $\mu=0.37$ and 0.5 respectively. Figure 5 a shows that the concentration of tensile $\sigma_{x x}$ is greater near the central valley than the tensile regime shown in figure $2 \mathrm{~b}$. The nature of $\sigma_{x z}$ and $\sigma_{z z}$ contours remains the same as in figure 2. But the maximum shear contours show less concentration on the central valley region. Figure 6 shows the stress contours for $\mu=0.5$ (liquid). Figure $6 \mathrm{~b}$ shows that $\sigma_{x x}$ 

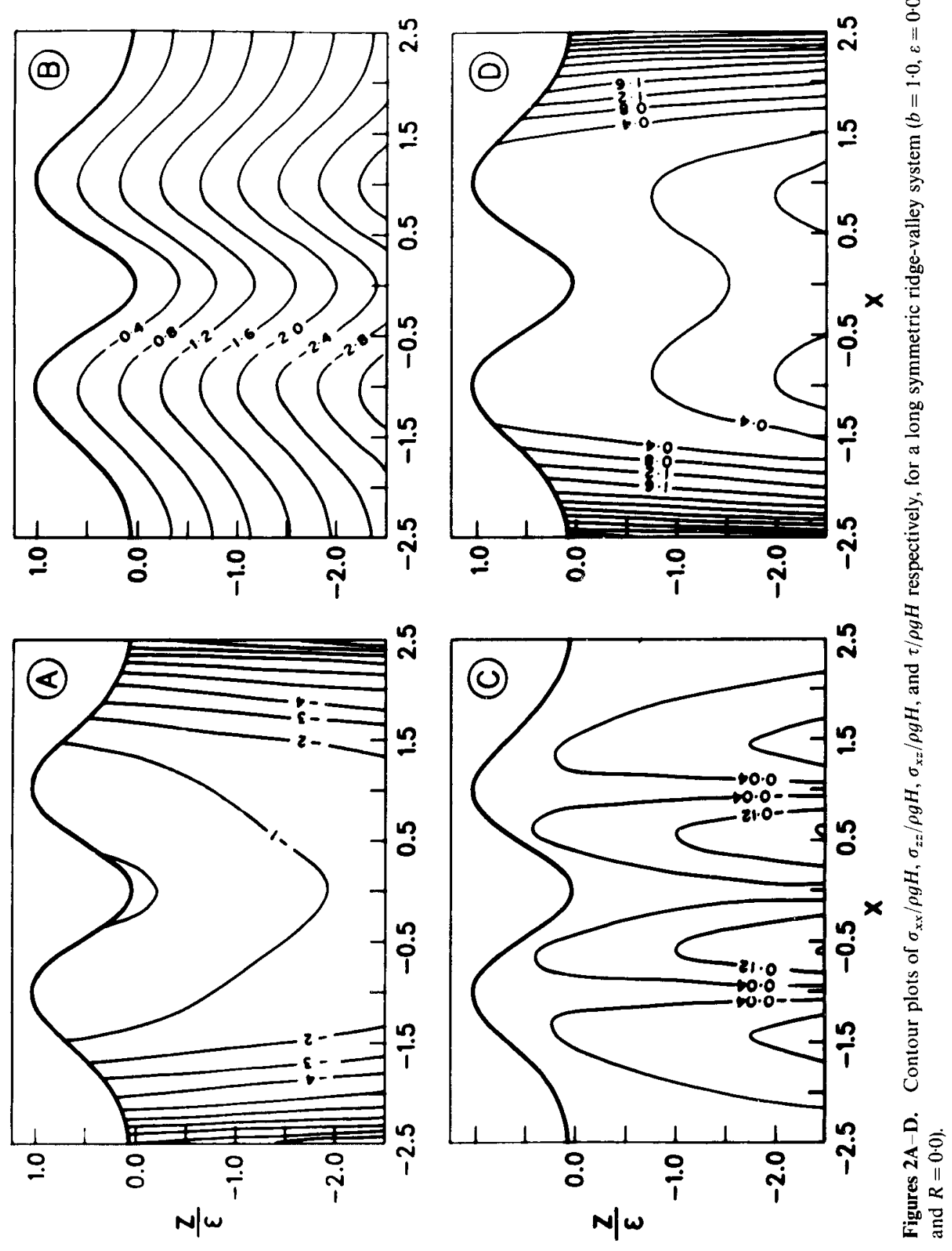

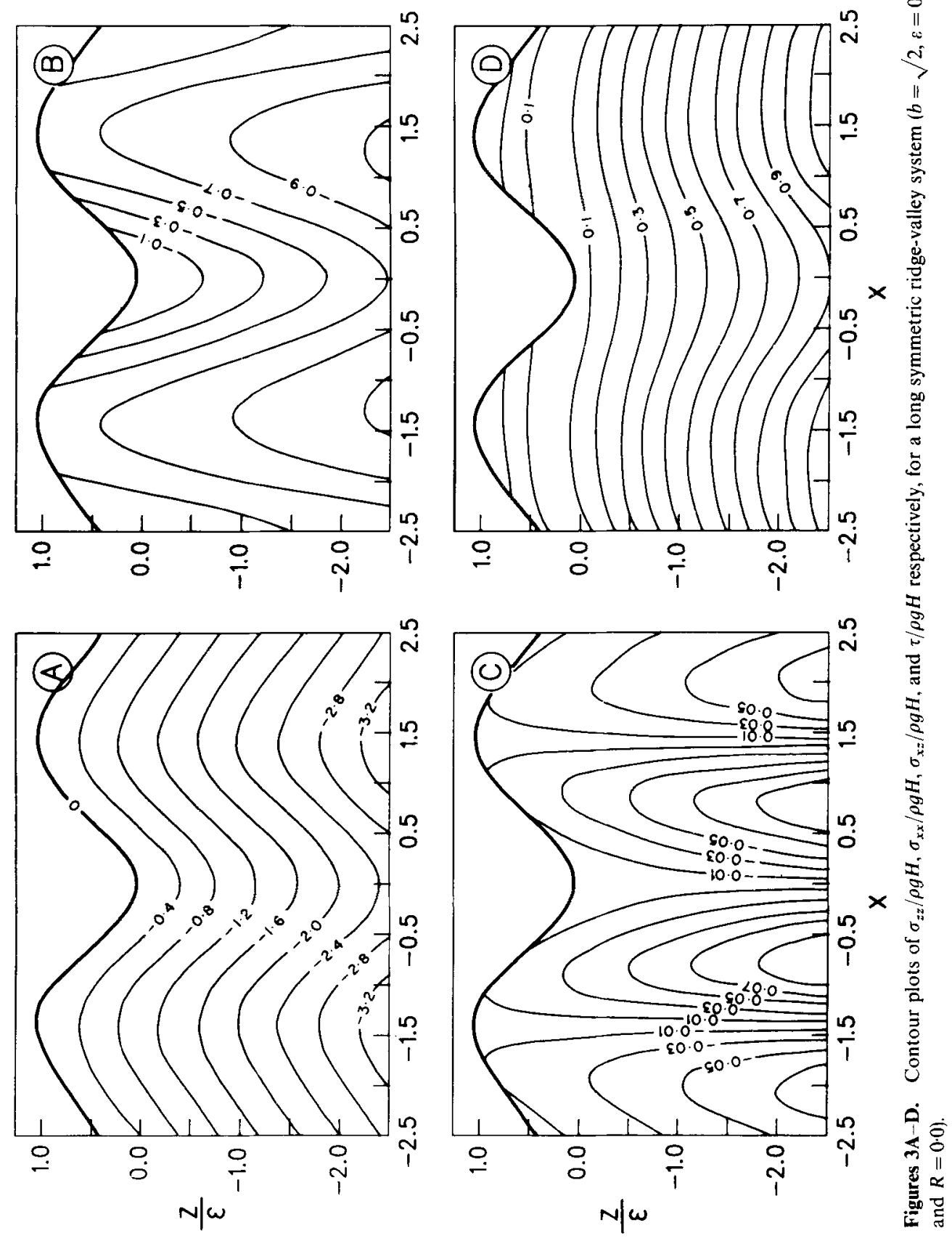

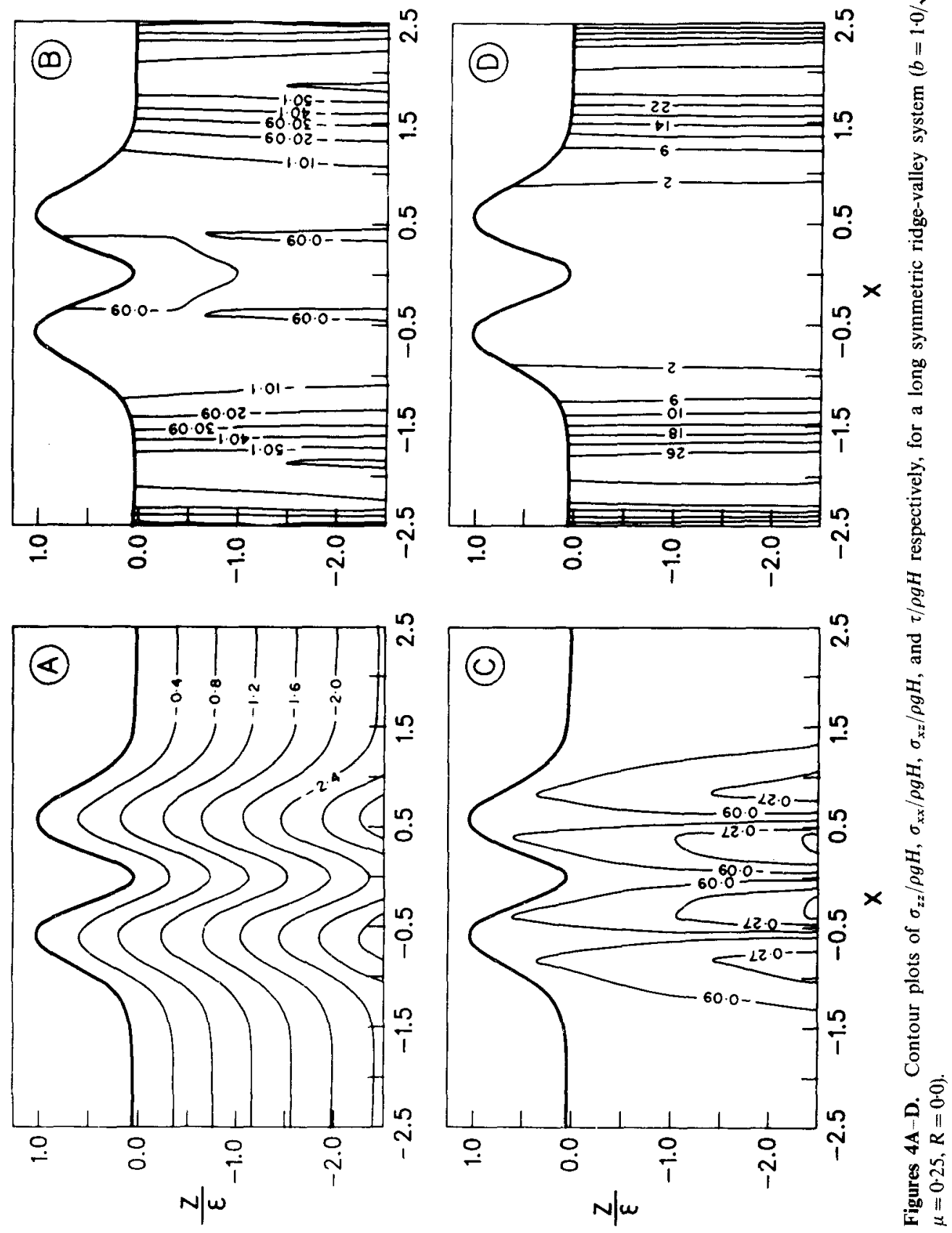

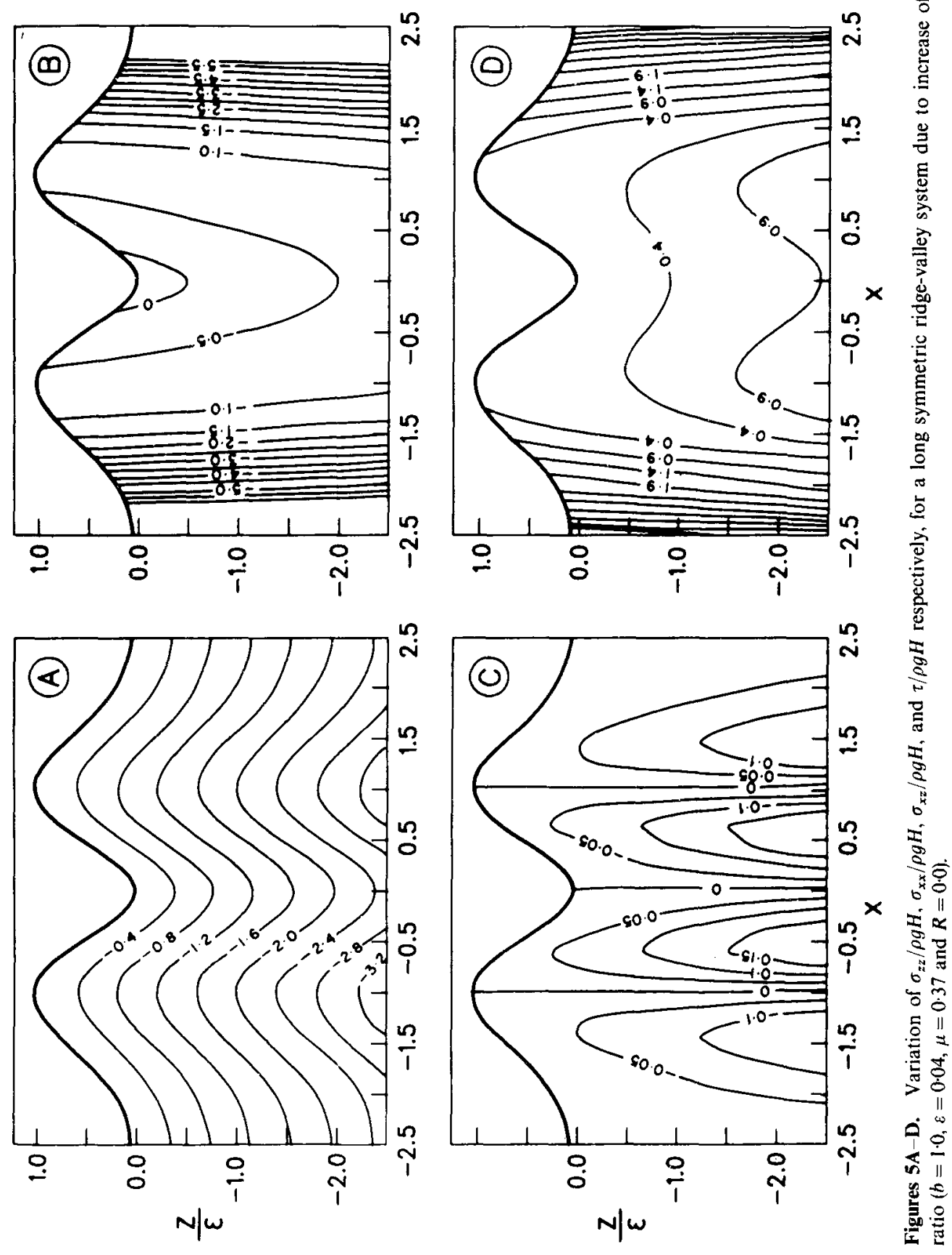

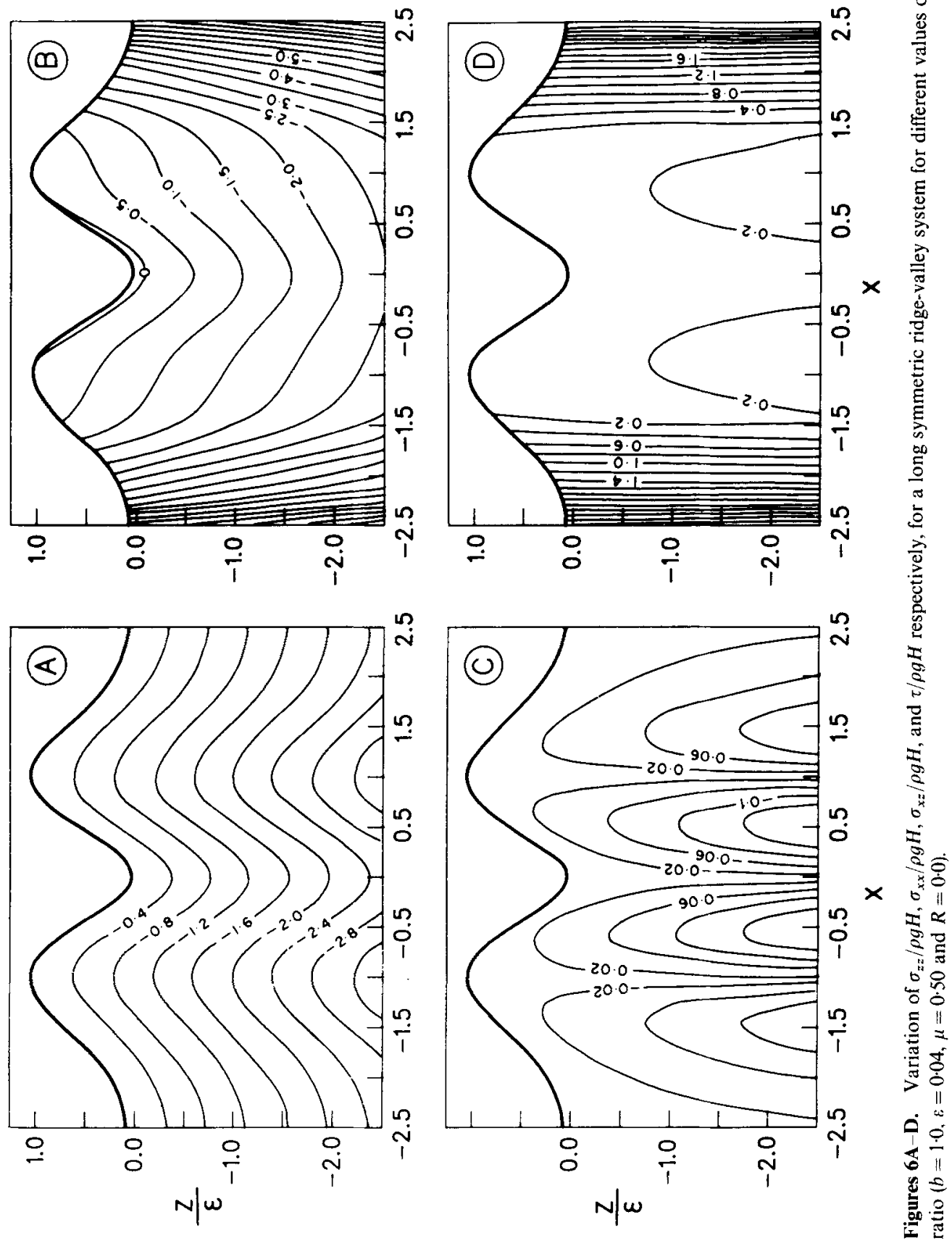

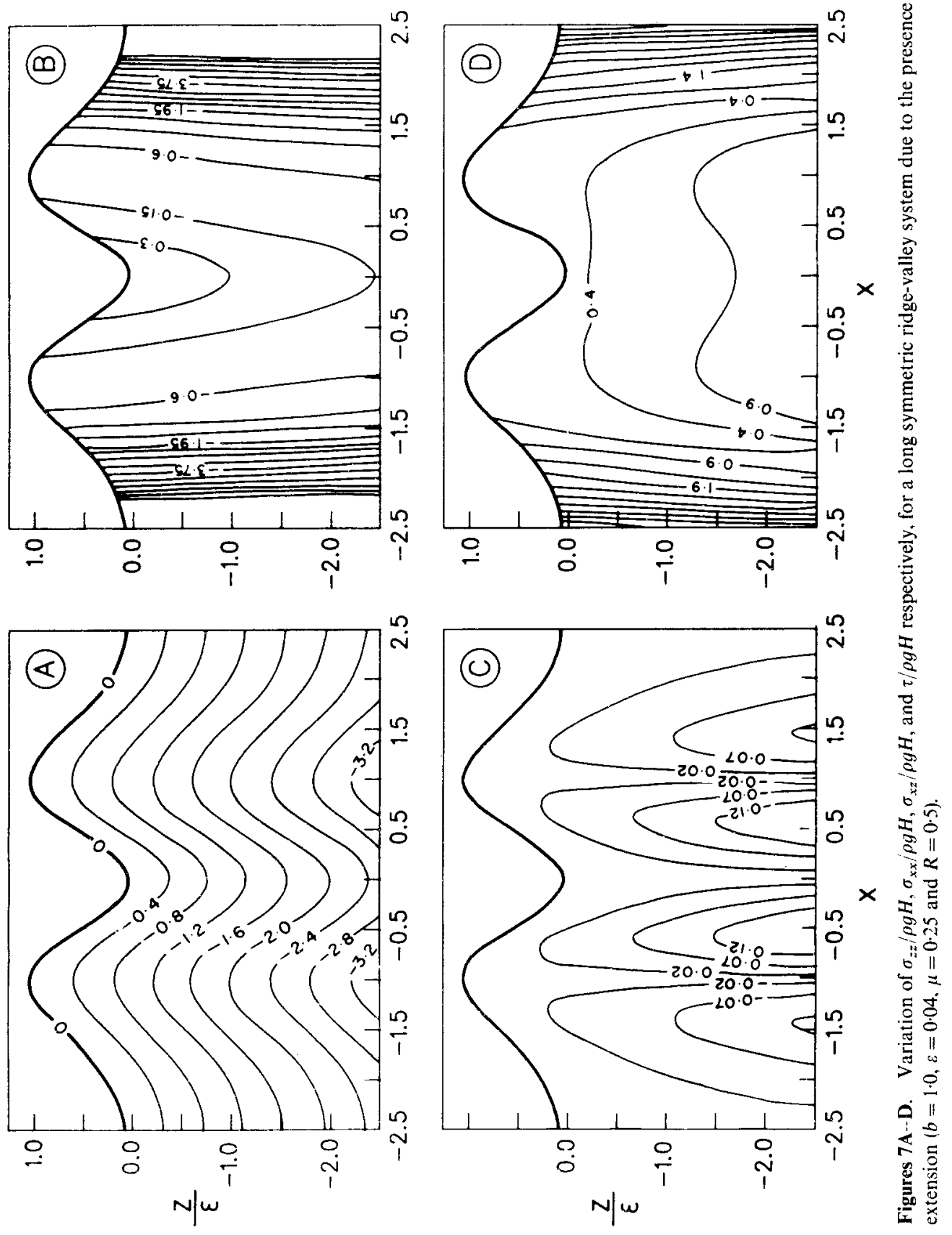

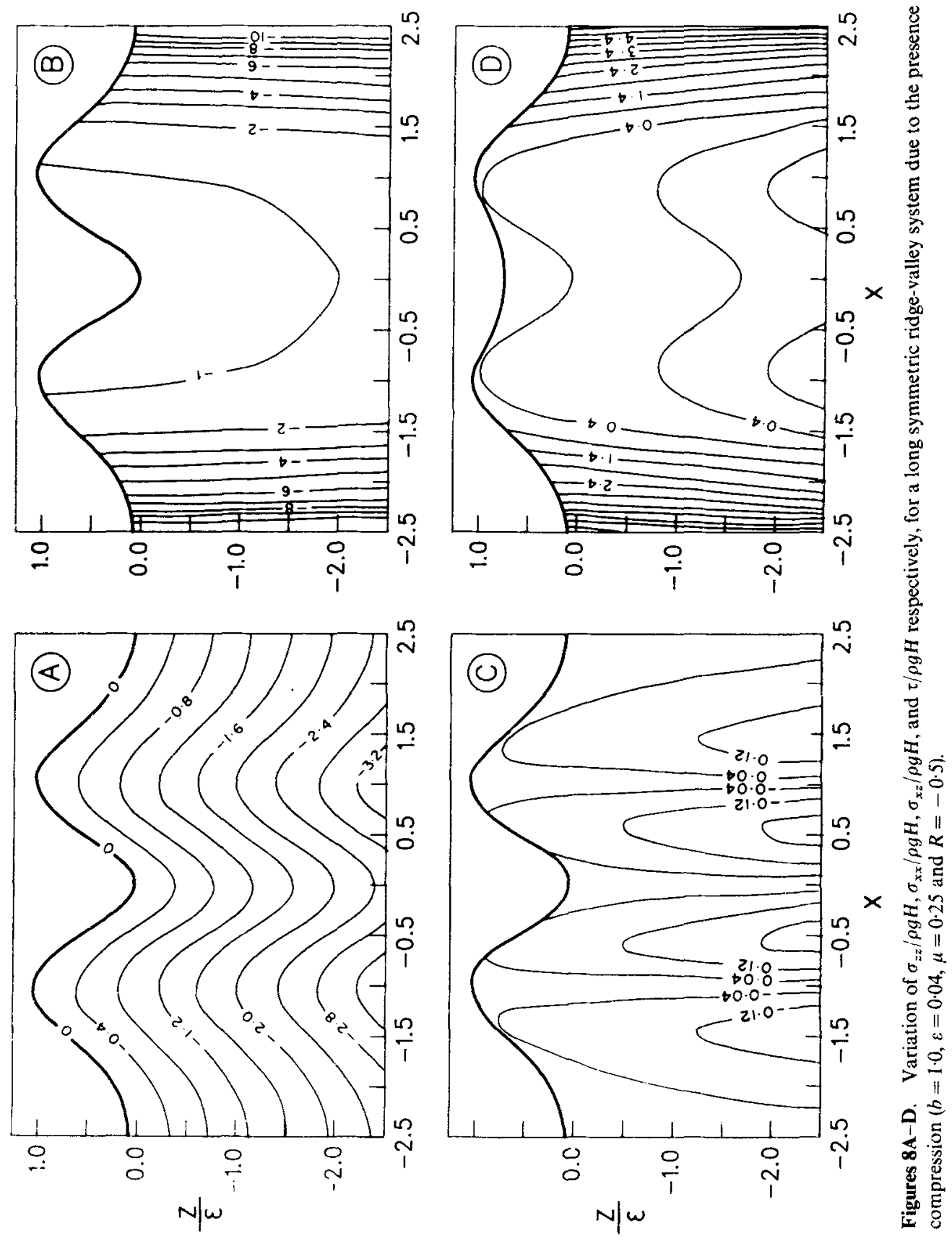
contours become narrower and more concentrated near the central valley region with zero value at the surface. Contours of $\sigma_{x z}$ and $\sigma_{z z}$ also show a trend similar to figures 2 and 5. But $\tau$ vanishes on the central valley and shows greater concentration at the outer flanks of the ridges.

\subsection{Effects of presence of the tectonic stresses}

The stress contours in the presence of a compressive $(R=-0.5)$ or tensile $(R=0.5)$ tectonic far-field stresses are shown in figures 7 and 8 respectively. Figure 7 shows relatively more sharp and compressed nature of stress contours than in figure 2 . A more or less flat and extended behaviour of stress contours is seen in figure 8 .

\section{Conclusions}

The following important results have been obtained from the preceding calculation:

(i) Magnitudes of stresses are of the order of $\rho g H$, where $H$ is the height of ridge or depth of the valley and vary with Poisson's ratio. In the far field, all stresses are tensional and become compressive at the ridges and tensional in the central valley region.

(ii) Stress regime under valley changes significantly due to the presence of two ridges on both sides of the valley compared to the case of isolated valley (Savage et al 1985) or the case of one valley and one ridge (McTigue and Mei 1981). The horizontal tensional regime under valley becomes shallower and narrower than in the above mentioned two cases.

(iii) Due to the presence of tensional regime in the valley between two ridges, the compressional stress values at the ridge crests are greater than in the case of isolated ridge or one valley and one ridge.

(iv) Non-zero compressive stresses, at or near ridge crests, persist even when surface slopes are small and decrease with increasing Poisson's ratio and become nearly zero at ridge crests (for $\mu=0.5$ ).

(v) The magnitude of tensional stresses decreases with increasing value of Poisson's ratio and becomes nearly zero at the central region of the ridge-valley systems.

In conclusion, we remark that these results would find applications in the study of seismicity and magma transport phenomena in the mid-crustal region and also in understanding stress regimes observed in hydraulic fracturing experiments near such structures.

\section{Acknowledgements}

Authors are grateful to Prof. D Guptasarma for his interest in this work. The lively discussions and valuable suggestions from Dr A O Gliko of the Institute of Physics of Earth, Moscow, USSR, visiting NGRI under the Indo-Soviet Long-Term Programme are gratefully acknowledged. The first author is thankful to the Department of Ocean Development for the award of a research fellowship. 


\section{References}

Akhpatelov D M and Ter-Martirosyan Z G 1971 The stressed state of ponderable semi-infinite domains; Armenian Acad. Sci. Mech. Bull. 24 33-40

Brotchie J F and Silvester R 1969 On crustal flexture; J, Geophys. Res. 74 5240-5252

Chen Y and Morgan W J 1990 A nonlinear rheology model for mid-ocean ridge axis topography; J. Geophys. Res. $9517583-17604$

Harrison J C 1976 Cavity and topographic effects in tilt and strain measurements; J. Geophys. Res. 81 $319-328$

Jaeger I C and Cook N G W 1976 Fundamentals of rock mechanics (New York: Halstead) p 356

Jeffreys H 1976 The Earth: its origin, history and physical constants (New York: Cambridge University Press) p 24

Lambeck K and Nakiboglu S M 1980 Seamount loading stress in the ocean lithosphere; J. Geophys. Res. $856403-6418$

McTigue D F and Mei C C 1981 Gravity-induced stresses near topography of small slope; J. Geophys. Res. 86 9268-9278

Panian J and Pilant W 1990 A possible explanation for foreland thurst propagation; J. Geophys. Res. 95 $8607-8615$

Savage W J and Swolfs H S 1986 Tectonic and gravitational stress in long symmetric ridges and valleys; J. Geophys. Res. 91 3677-3685

Savage W J, Swolfs H S and Powers P S 1985 Gravitational stress in long symmetric ridges and valleys; Int. J. Rock Mech. Min. Sci. Geomech. Abstr. 22 291-302

Strugel J R, Scheidegger A E and Grinshpan Z 1976 Finite element model of a mountain massif; Geology 4 439-442 